\title{
Coincident myelomeningocele and gastroschisis: report of 2 cases
}

\author{
Jason S. Hauptman, MD, PhD, Robert Bollo, MD, ${ }^{1}$ Rama Damerla, $\mathrm{PhD},{ }^{3}$ Brian Gibbs, $\mathrm{PhD},{ }^{3}$ \\ Cecilia Lo, PhD, ${ }^{3}$ Aviva Katz, MD, MA, ${ }^{4}$ and Stephanie Greene, MD ${ }^{5}$ \\ 1Department of Neurosurgery, Seattle Children's Hospital, Seattle, Washington; 2Department of Pediatric Neurosurgery, Primary \\ Children's Medical Center, Salt Lake City, Utah; ${ }^{3}$ Department of Embryology, University of Pittsburgh; and Departments of \\ ${ }^{4}$ Pediatric Surgery and ${ }^{5}$ Neurosurgery, Children's Hospital of Pittsburgh, Pennsylvania
}

\begin{abstract}
Myelomeningocele and gastroschisis, on their own, are both relatively common entities encountered in pediatric surgical care. Coexistence of these pathologies, however, is exceedingly rare. The authors report on 2 patients who presented with myelomeningocele and gastroschisis at birth. They obtained blood for whole-exome analysis for one of the patients and identified 3 mutations that could be related to the underlying anomalies: homozygous mutations in FAM171B and $A B C A 1$ and a hemizygous (X-linked) mutation in COL4A5. Of these, FAM171B and ABCA1 both have function that may be related to the underlying disease.
\end{abstract}

https://thejns.org/doi/abs/10.3171/2017.11.PEDS17540

KEY WORDS myelomeningocele; gastroschisis; Chiari II malformation; congenital

$\mathrm{W}$ HILE myelomeningocele and gastroschisis on their own are not uncommon pathologies encountered in neonatal care, their concurrent presentation is exceedingly rare. Gastroschisis, a right-sided, full-thickness defect of the anterior abdominal wall without a membrane, in which the bowel is in contact with amniotic fluid, occurs in approximately 1/4000 live births. ${ }^{6}$

Myelomeningocele, on the other hand, occurs on the order of 3.4 per 10,000 live births in the US. ${ }^{1}$ While other organ systems may be involved in gastroschisis cases in up to $30 \%$ of patients, ${ }^{2} \mathrm{CNS}$ involvement occurs less than $3 \%$ of the time. ${ }^{3}$ Furthermore, in a recent Texas registry, "spina bifida" was described in less than $1 \%$ of cases. We describe 2 patients with coincident myelomeningocele and gastroschisis. We were able to perform whole-exome sequencing in one of the patients, allowing us to identify several candidate gene mutations that may be involved in the pathogenesis of these anomalies.

\section{Case Reports}

\section{Case 1}

This male infant, who had a prenatal diagnosis of myelomeningocele and gastroschisis via fetal ultrasound, was born via cesarean section at 37 weeks' gestational age to a 17-year-old G1P1 mother with no family history of congenital anomalies. The birth weight was $3200 \mathrm{~g}$. Apgar scores were 6 at 1 minute and 8 at 5 minutes. Meconium staining was noted, and the patient was resuscitated, including endotracheal intubation. Prophylactic antimicrobial therapy with ampicillin and gentamycin were initiated, the gastroschisis and myelomeningocele were covered with sterile dressings, and the patient was transferred to the neonatal ICU with pediatric surgery and pediatric neurosurgery consultation. Neurological examination revealed hip flexor and quadriceps muscle weakness bilaterally, with spontaneous distal movement of both lower extremities and no foot deformity. The neural placode was flat, measuring approximately $4 \mathrm{~cm}$ in diameter. The head circumference was $37 \mathrm{~cm}$, with a flat anterior fontanelle and overriding coronal sutures. Based on prenatal discussions between the pediatric surgery and neurosurgery services, the surgical strategy was to proceed with gastroschisis closure first, followed by closure of the open neural tube defect the next day if the gastroschisis could be reduced, if the abdominal defect could be closed, and if the patient remained stable.

On the 1st day of life, the patient was brought to the operating room by the pediatric surgery team for skin clo- 
sure of the gastroschisis. He was positioned supine with a custom donut pillow. The myelomeningocele was covered with petroleum dressing and sterile gauze and suspended within the donut to avoid pressure on the neural placode. The abdomen was carefully inspected, and the majority of intestinal viscus was noted to be extraperitoneal. The abdomen was prepared and draped in sterile fashion, and the bowel was carefully dissected and reduced into the peritoneum, without changes in peak inspiratory or mean airway pressures. The fascia was left open, and the skin was closed with interrupted vertical mattress sutures.

On the 2nd day of life, the patient was brought back to the operating room for neurosurgical closure of the myelomeningocele. He was positioned prone with the chest and abdomen supported by gel rolls with care to avoid pressure on the abdominal surgical wound. The myelomeningocele was repaired in the typical fashion, including imbrication of the placode, a primary dural closure, and a multilayered soft-tissue closure. This occurred without complication.

The following day, total parental nutrition was initiated. An echocardiogram demonstrated a small patent ductus arteriosus with right-to-left shunting, and a small atrial right-to-left shunt, with normal ventricular function and no evidence of ventricular septal deficits. A CT scan of the head without contrast obtained the same day revealed moderate, symmetric ventriculomegaly. On the 5th day of life, a ventriculoatrial CSF shunt was inserted for the treatment of hydrocephalus due to a rapidly increasing head circumference and clinical signs of raised intracranial pressure. The peritoneum was avoided in the context of the recent surgery. Shunt placement was complicated by a left temporal intraparenchymal hemorrhage diagnosed on routine postoperative head CT scanning the next day, attributed to rapid intracranial decompression. Serial radiographic surveillance with ultrasound demonstrated progressive resolution of the hematoma, but clinical seizure activity was observed on the 8th day of life. Phenobarbital was initiated, and findings of routine electroencephalography on the 9th day of life were normal. The patient was transitioned to enteral feeding on the 15th day of life, and discharged from the neonatal ICU on the 28th day of life, feeding well without supplemental nutrition.

\section{Case 2}

This male infant was born at $363 / 7$ weeks to a 29-yearold G4P3 mother via spontaneous rupture of the membranes and subsequent vaginal delivery. The child had prenatal diagnoses of gastroschisis, myelomeningocele, and Chiari II malformation with ventriculomegaly by ultrasound and fetal MRI (Fig. 1). There had been a plan for induction of labor at 38 weeks, but the mother presented to the hospital in preterm labor and progressed to delivery. Aside from the mother testing positive for group B Streptococcus, which was treated shortly after delivery, there were no significant maternal risk factors. The baby was born at $3360 \mathrm{~g}$ weight (75\%), $47 \mathrm{~cm}$ length (40\%), and $31 \mathrm{~cm}$ occipitofrontal circumference (14\%). The patient's Apgar scores were 1 at 1 minute, 3 at 5 minutes, and 5 at 10 minutes. Because of respiratory distress, the infant required intubation and mechanical ventilation at $12 \mathrm{~min}-$ utes of life.

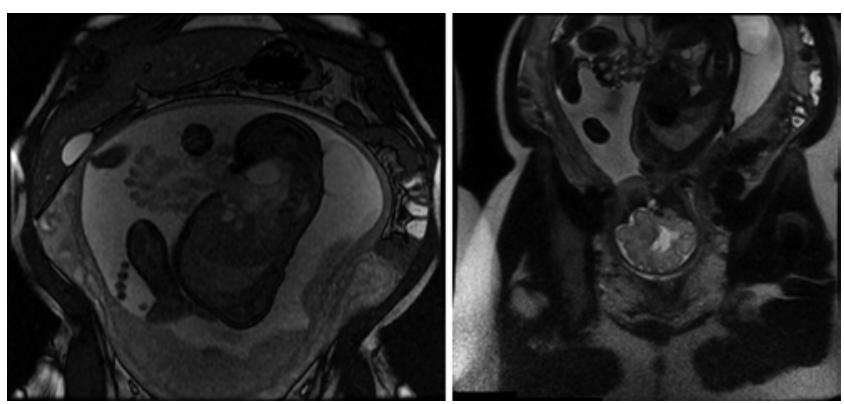

FIG. 1. Case 2. Prenatal MR images showing coincidental gastroschisis and lumbosacral myelomeningocele as well as ventriculomegaly.

On examination, the child had a large defect in the anterior abdominal wall with proximal bowel dilation, although it was pink throughout (Fig. 2). The child also had a $6 \times 6-\mathrm{cm}$ ruptured lumbosacral myelomeningocele. Other than weak hip flexion, the lower extremities were flaccid. Empirical ampicillin and gentamycin were initiated, hydration according to a gastroschisis protocol was begun, and a moist sterile covering was applied to the myelomeningocele. On the 1st day of life, the infant was brought to the operating room for an uncomplicated primary closure of the gastroschisis. The gastroschisis was closed in a similar fashion to that in case 1, with the exception being that there was a primary fascial closure in addition to skin closure. Prior to this operation, the child was carefully positioned in a custom donut pillow with the myelomeningocele suspended within the donut. To allow for adequate antibiotic coverage and recovery, the myelomeningocele repair was deferred until the following day. In the interim, a head ultrasound showed no evidence of ventriculomegaly.

On the 2nd day of life, the infant was taken to the operating room for repair of the myelomeningocele. The myelomeningocele was closed in typical fashion, with imbrication of the placode, an inlay synthetic dural patch graft, and a multilayered soft-tissue closure. Postoperative MRI demonstrated mild ventricular enlargement and a Chiari II malformation, without evidence of syringomyelia. The child recovered well from both surgeries, was extubated, was able to tolerate enteral feeding by day 9 of life, and was discharged from the hospital 15 days after birth with-

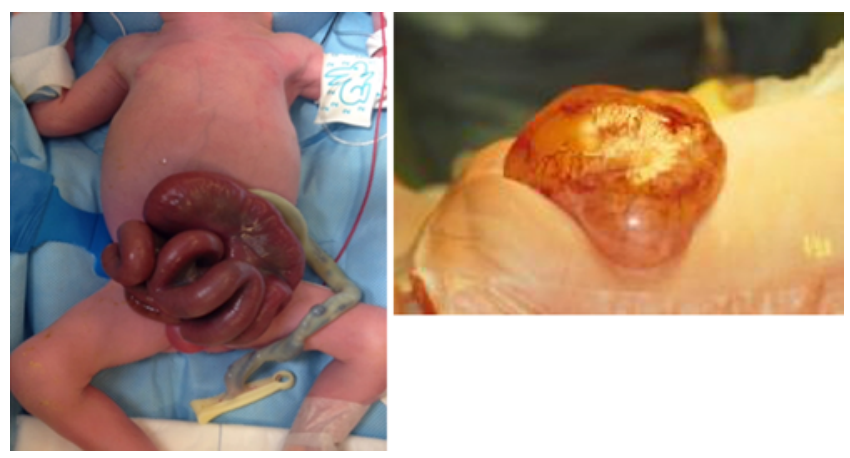

FIG. 2. Case 2. Intraoperative photographs showing gastroschisis (left) and lumbosacral myelomeningocele (right). Figure is available in color online only. 
out further complications. During the hospitalization, biweekly ultrasound studies failed to reveal significant ventricular enlargement, and the head growth velocity was not abnormal. On last follow-up at 3 months of age, the patient has an L-4 functional level with weakness in dorsiflexion and plantar flexion. He undergoes catheterization intermittently and requires an aggressive bowel regimen. His head circumferences have followed expected growth curves, and imaging has failed to reveal a significant increase in ventricular size. He has not required a shunt.

\section{Discussion}

Myelomeningocele and gastroschisis result from failure of closure of the neuroectodermal and endodermal tubes, respectively. ${ }^{14}$ While concurrent existence of these 2 anomalies is rare, it has been described before. ${ }^{6,15}$ Little has been described as to the potential etiology of these intersecting problems. In this report, we attempt to begin to answer this question through whole-exome sequencing of one of the affected patients.

The patient whose blood was obtained for wholeexome analysis (case 2) presented with myelomeningocele with ventriculomegaly, Chiari II malformation, gastroschisis, and atrial septal defect. Whole-exome sequencing of the patient identified homozygous mutations in $F A M 171 B$ and $A B C A 1$ and a hemizygous (X-linked) mutation in COL4A5 (Table 1). COL4A5, a type IV collagen within the basement membrane, is thought to play a role in lamination and axonal connectivity in the developing brain. ${ }^{18}$ COL4A5 mutations have been linked with Alport syndrome, a genetic disorder characterized by hearing impairment, ocular defects, and progressive glomerulonephritis leading to renal failure. ${ }^{10}$

FAM171B is an uncharacterized protein with unknown function. One group of authors has reported a mutant mouse with gastroschisis harboring a mutation in Slit3 and an additional point mutation in Fam171A1, a related family member that has $35 \%$ amino acid similarity to $F A M 171 B .{ }^{11}$ In addition to gastroschisis, this mutant mouse presented with double-outlet right ventricle with an atrioventricular septal defect, ventricular septal defects, and ventricular noncompaction (http://www.informatics. jax.org/allele/key/842100).

ABCA1, ATP-binding cassette transporter 1, is a cholesterol transporter and is associated with Tangier syndrome and familial high-density lipoprotein deficiency, 2 disorders characterized by reduced or deficient high-density lipoprotein cholesterol. ABCA1 also has been shown to play a role in maternal-fetal cholesterol transfer, a process disrupted in Smith-Lemli-Opitz syndrome, a genetic

TABLE 1. Possible pathogenic variants from whole-exome sequencing analysis

\begin{tabular}{ccccc}
\hline \multicolumn{1}{c}{ Gene } & Type & Dosage & Chromosome & Position \\
\hline FAM171B & $\begin{array}{c}\text { In-frame in- } \\
\text { sertion }\end{array}$ & Homozygous & 2 & 187559029 \\
\hline ABCA1 & Splicing & Homozygous & 9 & 107556793 \\
\hline COL4A5 & Stopgain & Hemizygous & $\mathrm{X}$ & 107936016 \\
\hline
\end{tabular}

disorder characterized by multiple congenital abnormalities due to disruption of sonic hedgehog (Shh) signaling.,4 Significantly, the disruption of Shh signaling is also well described to play a role in the pathophysiology of neural tube defects, ${ }^{13}$ VACTERL association,,${ }^{9,17}$ and recently also implicated in ventral body wall closure defects, including omphalocele. ${ }^{5,7,12}$ In vivo, amniotic fluid and serum levels of Shh are linked to neural tube defects. ${ }^{16}$ We therefore speculate that the predicted splicing mutation in $A B C A I$ could perturb Shh signaling relate to the intersecting failure of closure of the ventral wall and neural tube.

Gastroschisis with myelomeningocele is an uncommon clinical entity, although one that may provide insights into underlying genetic anomalies associated with them. In one of our cases, whole-exome sequencing identified potential mutations that might have resulted in the clinical entities observed here. Interestingly, the anomalies do not appear to be linked to folate demethylation, a surprising finding given the presumed etiology of spinal dysraphism. ABCA1, known to be involved in Shh signaling, may account for neural tube and ventral abdominal wall defects. FAM171B, a protein for which the function still has not been demonstrated, has a related protein (FAM171A1) shown to be involved in gastroschisis and cardiac anomalies in laboratory animals. Future work will focus on understanding the role of these proteins in vivo and in vitro with regard to the pathophysiology of neural tube and ventral wall defects.

\section{References}

1. Au KS, Tran PX, Tsai CC, O’Byrne MR, Lin JI, Morrison $\mathrm{AC}$, et al: Characteristics of a spina bifida population including North American Caucasian and Hispanic individuals. Birth Defects Res A Clin Mol Teratol 82:692-700, 2008

2. Benjamin B, Wilson GN: Anomalies associated with gastroschisis and omphalocele: analysis of 2825 cases from the Texas Birth Defects Registry. J Pediatr Surg 49:514-519, 2014

3. Benjamin B, Wilson GN: Registry analysis supports different mechanisms for gastroschisis and omphalocele within shared developmental fields. Am J Med Genet A 167A:2568-2581, 2015

4. Cooper MK, Wassif CA, Krakowiak PA, Taipale J, Gong R, Kelley RI, et al: A defective response to Hedgehog signaling in disorders of cholesterol biosynthesis. Nat Genet 33:508513,2003

5. Daane JM, Downs KM: Hedgehog signaling in the posterior region of the mouse gastrula suggests manifold roles in the fetal-umbilical connection and posterior morphogenesis. Dev Dyn 240:2175-2193, 2011

6. Dharmraj M, Verma AP: Gastroschisis associated with lower limb and spinal congenital anomalies. J Clin Neonatol 1:217-220, 2012

7. Fujiwara N, Duess J, Puri P, Thompson J: Disturbance of SHH signalling pathway during early embryogenesis in the cadmium-induced omphalocele chick model. Pediatr Surg Int 29:165-170, 2013

8. Gruchy N, Bigot N, Jeanne Pasquier C, Read MH, Odent $\mathrm{S}$, Galera $\mathrm{P}$, et al: Involvement and alteration of the Sonic Hedgehog pathway is associated with decreased cholesterol level in trisomy 18 and SLO amniocytes. Mol Genet Metab 112:177-182, 2014

9. Kim PCW, Mo R, Hui CC: Murine models of VACTERL syndrome: role of sonic hedgehog signaling pathway. J Pediatr Surg 36:381-384, 2001 
10. Kruegel J, Rubel D, Gross O: Alport syndrome-insights from basic and clinical research. Nat Rev Nephrol 9:170178,2013

11. Li Y, Klena NT, Gabriel GC, Liu X, Kim AJ, Lemke K, et al: Global genetic analysis in mice unveils central role for cilia in congenital heart disease. Nature 521:520-524, 2015

12. Matsumaru D, Haraguchi R, Miyagawa S, Motoyama J, Nakagata N, Meijlink F, et al: Genetic analysis of Hedgehog signaling in ventral body wall development and the onset of omphalocele formation. PLoS One 6:e16260, 2011

13. Murdoch JN, Copp AJ: The relationship between sonic Hedgehog signaling, cilia, and neural tube defects. Birth Defects Res A Clin Mol Teratol 88:633-652, 2010

14. Sadler TW, Feldkamp ML: The embryology of body wall closure: relevance to gastroschisis and other ventral body wall defects. Am J Med Genet C Semin Med Genet 148C:180-185, 2008

15. Singal R, Garg LN, Singal RP, Gupta S, Shahi SR, Singal S, et al: Omphalocele and gastroschisis associated with multiple congenital abnormalities. J Med Life 4:295-296, 2011

16. Tsurubuchi T, Ichi S, Shim KW, Norkett W, Allender E, Mania-Farnell B, et al: Amniotic fluid and serum biomarkers from women with neural tube defect-affected pregnancies: a case study for myelomeningocele and anencephaly: clinical article. J Neurosurg Pediatr 12:380-389, 2013

17. Vaze D, Mahalik S, Rao KL: Novel association of VACTERL, neural tube defect and crossed renal ectopia: sonic hedgehog signaling: a point of coherence? Congenit Anom (Kyoto) 52:211-215, 2012

18. Xiao T, Staub W, Robles E, Gosse NJ, Cole GJ, Baier H: Assembly of lamina-specific neuronal connections by slit bound to type IV collagen. Cell 146:164-176, 2011

\section{Disclosures}

The authors report no conflict of interest concerning the materials or methods used in this study or the findings specified in this paper.

\section{Author Contributions}

Conception and design: Hauptman, Bollo, Greene. Acquisition of data: Hauptman, Bollo, Damerla, Katz, Greene. Analysis and interpretation of data: Hauptman, Bollo, Damerla, Gibbs, Lo, Greene. Drafting the article: Hauptman, Bollo, Damerla, Lo. Critically revising the article: Hauptman, Bollo, Lo, Greene. Reviewed submitted version of manuscript: Hauptman, Bollo, Damerla, Lo, Katz, Greene. Approved the final version of the manuscript on behalf of all authors: Hauptman.

\section{Correspondence}

Jason S. Hauptman: Seattle Children's Hospital, Seattle, WA. jason.hauptman@seattlechildrens.org. 